\title{
Connecting high-redshift galaxy populations through observations of local Damped Lyman Alpha dwarf galaxies
}

\author{
Regina E. Schulte-Ladbeck \\ Department of Physics and Astronomy, University of Pittsburgh, \\ Pittsburgh, PA 15260, USA \\ email: rsl@pitt.edu
}

\begin{abstract}
I report on observations of the $\mathrm{z}=\mathrm{t} 0.01$ dwarf galaxy SBS1543 +593 which is projected onto the background QSO HS1543+5921. As a star-forming galaxy first noted in emission, this dwarf is playing a pivotal role in our understanding of high-redshift galaxy populations, because it also gives rise to a Damped Lyman Alpha system. This enabled us to analyze, for the first time, the chemical abundance of $\alpha$ elements in a Damped Lyman Alpha galaxy using both, emission and absorption diagnostics. We find that the abundances agree with one another within the observational uncertainties. I discuss the implications of this result for the interpretation of high-redshift galaxy observations. A catalog of dwarf-galaxy-QSO projections culled from the Sloan Digital Sky Survey is provided to stimulate future work.
\end{abstract}

Keywords. line: formation, methods: data analysis, techniques: spectroscopic, galaxies: ISM, galaxies: abundances, galaxies: dwarf, galaxies: starburst, quasars: absorption lines

\section{The Problem}

We now know a wealth of galaxy populations at high redshifts. Damped Lyman Alpha (DLA) systems are identified from the strong neutral Hydrogen gas absorption they cause in background QSO spectra, while Lyman Break Galaxies, Lyman Alpha Emitters, and Gamma Ray Burst (GRB) host galaxies are discovered through emission from their stars and ionized gas. What we would yet like to understand is how these populations relate to each other, and to the galaxies we see today. While the study of DLA systems has made great contributions to our empirical knowledge of the chemical evolution of galaxies, unfortunately, despite decades of observational searches it has proven extremely difficult to detect the host galaxies directly in emission (cf. Dessauges-Zavadsky, this volume). Our understanding of high redshift galaxies remains biased by how they were selected, via absorption or emission.

Figure 1 shows $\alpha$ element abundances for star-forming galaxies (SFG) and HII regions derived from emission lines (these are $\mathrm{O}$ abundances), as well as for DLA systems determined from absorption lines (O, S, or Si abundances). This figure illustrates several key points which have entered in the debate over the abundances of emission- versus absorption-selected systems. First, emission diagnostics tend to dominate the low-redshift abundance data while absorption diagnostics are still the method of choice for deriving high-redshift abundances. And second, there appears to be very little overlap in the $\alpha$ element abundances derived using emission versus absorption techniques. What does that mean for the galaxy populations probed? 


\section{Proposed Solutions}

Four solutions to this problem have been thought of at this time. They are briefly summarized below.

Diagnostics Hypothesis. The diagnostics hypothesis posits that the absorption lines give lower metallicities than the emission lines because this is an inherent difference in the diagnostics. Verifying or falsifying this hypothesis has been the focus of our work, and will be the main topic of this paper.

Different Radial Biases Hypothesis. The different radial biases hypothesis predicts that lower abundances will be measured in absorption than in emission if the negative radial abundance gradient observed in local spirals (Searle 1971) holds for DLAs in general. Starformation tends to occur in the centers of galaxies; QSO sightlines intercept foreground systems at any radii where the HI column density is above DLA threshold, thus, on average, they will be probing larger radii.

Distinct Populations Hypothesis. The distinct population hypothesis postulates that low abundances for DLAs result if they are comprised of low-mass or dwarf (proto-)galaxies (Haehnelt et al. 1998). Mass-metallicity relations have now been shown to exist out to redshifts of about 3 (cf. Mannucci, Lee, this volume).

Dust Obscuration Bias Hypothesis. A metal-rich interstellar medium tends to be dusty as well (cf. Hunter, Spaans, this volume). The dust obscuration bias hypothesis predicts low abundances for DLAs result if optically selected QSO samples are biased toward QSOs with little foreground extinction (Ostriker \& Heisler 1984).

\section{Testing the Diagnostics Hypothesis}

The emission-line technique is based on the measurement of forbidden-line fluxes. Line fluxes need to be corrected for stellar and foreground dust absorptions. We then determine electron temperature, density, and ionic abundances, which, with appropriate ionization corrections, lead to element abundances. When the temperature cannot be derived, and for high-redshift galaxies, we often use a strong-line method to evaluate abundances rather than the direct or Te method. The emission-line technique for deriving abundances is described more fully in Stasinska (this volume).

The absorption-line technique is based on the measurement of absorption-line profiles. Their optical depths lead to ionic column densities. Dust depletion and ionization corrections must be considered; then we determine element abundances. When high-resolution spectra are not available, such as is the case with FUSE and HST data of DLAs, we use the curve-of-growth method to estimate an ion's column density.

What do we know about whether the physics of the line diagnostics works for astronomical objects? Williams et al. (2008) have recently compared the abundances of planetary nebulae measured in emission with the abundances measured in absorption toward their central stars. They find good agreement between forbidden emission-line and absorption-line abundances for a wide range of elements.

What do we know about how the diagnostics work on the scale of galaxies? And wouldn't the abundances depend on whether and how well the different phases of the interstellar medium in which they are measured are mixed? In the Galaxy, HII regions abundances interpolated for the distance of the solar circle (Deharveng et al. 2000) agree very well with abundances in the local ISM measured on sightlines toward nearby hot stars (Moos et al. 2002). This leads to the expectation that emission and absorption diagnostics should also give concordant abundances when applied to other galaxies. 


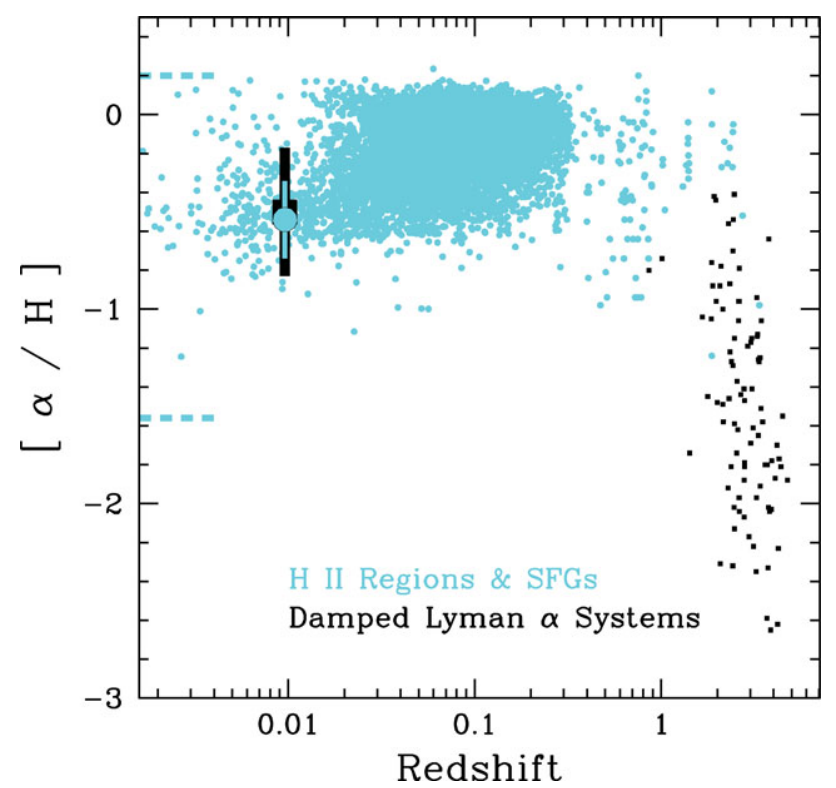

Figure 1. Alpha element abundances for SFGs and DLAs (adapted from König et al. 2006). The dashed lines indicate the highest and lowest metallicities measured in the local galaxy population. Our measurements for the $[\mathrm{O} / \mathrm{H}]_{I I}$ and $[\mathrm{S} / \mathrm{H}]_{I}$ abundances of SBS1543 +593 are shown as well.

\subsection{Test Designs}

There are two experimental designs that have been applied to the study of emissionand absorption-based abundances in external galaxies. The first method uses an internal starcluster or starburst within a galaxy (cf. contributions by Lebouteiller, Aloisi, and Thuan in this volume). The advantage of doing so is that the HII and the HI gas are both on the sightline to the starcluster. The disadvantage is that the starcluster may contain thousands of hot stars and is spatially extended; it also lies behind a complex foreground screen. The size of the aperture used for the absorption spectroscopy usually results in an integration over these many, diverse sightlines. This is a potential problem that could be fixed if a single hot star could be picked out from the cluster, but that has not been possible due to the high spatial resolution that would be required.

The second method employs the classical absorption technique by using a QSO as the background source. The advantage is that the background source is clearly a point source. The disadvantage is that the probability of finding a QSO sightline that is aligned with an HII region in a foreground galaxy is extremely small. Recall it has been hard to find DLA galaxies at all. We therefore cannot, at this time, measure the HII abundances on the same sightlines as the HI abundances. This could be fixed if we could find a QSO behind an HII region. A possible workaround is to use an HII region in a dwarf galaxy, because, to the best of our knowledge, dwarf galaxies do not exhibit radial abundance gradients (cf. Stasinska, this volume). For this to work, one has to find suitable dwarf DLA galaxy-QSO projections. Until recently, there was only one known case.

\section{2. $S B S 1543+593$}

The alignment of SBS1543+593 ( $\mathrm{z}=0.0096)$ with the QSO HS1543+ 5921 was discovered by Reimers \& Hagen (1998). Previously, the galaxy had been thought of as having a Seyfert nucleus. The discovery that the galaxy intercepts the QSO at a mere $0.5 \mathrm{kpc}$ 
finally enabled the test of diagnostics hypothesis. Bowen et al. (2001) found that the sightline to the QSO gives rise to a DLA. Schulte-Ladbeck et al. (2004) classified the galaxy an Sdm, determined its absolute B magnitude $(-16.8)$ and estimated its starformation rate $\left(0.006 \mathrm{M}_{\odot} \mathrm{yr}^{-1}\right)$. We also derived $12+\log (\mathrm{O} / \mathrm{H})_{I I}$ is $8.2 \pm 0.2$, or $[\mathrm{O} / \mathrm{H}]_{I I}$ is $-0.54 \pm 0.2$. In Schulte-Ladbeck et al. (2005) we also derive a Sulphur abundance in emission, and determine $[\mathrm{S} / \mathrm{H}]_{I I}$ is $-0.27 \pm 0.3$. Our analysis of HST spectra, on the other hand, yielded $[\mathrm{O} / \mathrm{H}]_{I}>-2.14$ (the line tends to be saturated) and $[\mathrm{S} / \mathrm{H}]_{I}=-0.50 \pm 0.33$ (this may be a lower limit as the lines were unresolved and may therefore contain hidden saturated components). The $[\mathrm{O} / \mathrm{H}]_{I I}$ and $[\mathrm{S} / \mathrm{H}]_{I}$ data are plotted in Fig. 1.

\subsection{Result}

Our experiment indicates that the emission- and absorption-derived $\alpha$ element abundances of SBS1543+593 agree within the errors. The diagnostics hypothesis has been falsified. In other words, the hypothesis that QSO absorption lines give lower abundances than HII emission lines for (proto-)galactic systems is not true.

\subsection{Verification}

Analysis of the same HST data by Bowen et al. (2005) resulted in $[\mathrm{O} / \mathrm{H}]_{I}>-0.9$ and $[\mathrm{S} / \mathrm{H}]_{I}=-0.41 \pm 0.06$, in good agreement with Schulte-Ladbeck et al. (2004, 2005). There has been no independent confirmation of the ionized gas abundances in SBS1543 +593 .

\subsection{Implications}

Our result indicates that abundances derived via HII-region emission lines and QSO absorption lines are directly comparable. The difference between the abundances measured in SFGs and DLAs is real. Our result also implies that the neutral gas of SBS1543+593, which extends out to 15 times its optical radius (Rosenberg et al. 2006), has the same abundance as the ionized gas found within the confines of the galaxy.

Abundances for high-redshift GRB host galaxies are now routinely determined from absorption lines. This is analogous to the QSO experiment since the GRB is a point source. Swift recently discovered a wealth of low-redshift GRBs. With HST lacking UV spectroscopic ability, the abundances of the host galaxies have been detemined using emission lines. Savaglio (this volume) shows an abundance-redshift plot for GRB host galaxies, combining the low-redshift emission abundances with the high-redshift absorption abundances, to discuss trends in metallicity evolution of GRBs. Our result indicates that this is indeed a valid comparison.

\section{Future Work: A Catalog of Dwarf Galaxies on QSO Sightlines}

It would be helpful to obtain confirmation of our result through observation of more dwarf galaxies on QSO sightlines. Table 1 is a catalog of SDSS dwarf galaxy $\left(\mathrm{M}_{B}>-18\right)$ \& QSO pairs from B. Cherinka's thesis project. It gives the SDSS galaxy, QSO name, redshifts, galaxy luminosity ( $\mathrm{k}$ - and foreground absorption corrected, in the $\mathrm{g}$ band), and ratio of impact parameter to galaxy r-band radius. Based on the strength of their CaII or NaI QSO absorption lines, potential candidates for DLA galaxies are SDSS $\mathrm{J} 032803.11+002055.1$, J125700.31 + 010143.3 (=UGC 8066), J170330.32 + 240330.8, and $\mathrm{J} 221216.89+003243.9$. It should be noted that the SDSS QSO spectra are of quite low $\mathrm{S} / \mathrm{N}$, therefore, our absorption line measurements are quite uncertain. UGC 8066, an LSB galaxy with $\log \mathrm{M}_{H I}\left[\mathrm{M}_{\odot}\right]=9.29$ and $\mathrm{M}_{H I} / \mathrm{L}_{B} \approx 2$ (Burkholder et al. 2001), is perhaps our best candidate. 
Table 1. Dwarf Galaxies on QSO sightlines in SDSS

\begin{tabular}{|c|c|c|c|c|c|}
\hline SDSS Galaxy Name & $\mathbf{z}_{\mathrm{G} \mathrm{A} \mathrm{L}}$ & SDSS QSO Name & $\mathrm{z}_{\mathrm{Q}} \mathrm{S}$ O & $\begin{array}{l}\mathbf{L}_{\mathbf{g}^{*}, \mathbf{f}} \\
{\left[\mathbf{L}_{\mathrm{g}}^{*}\right]}\end{array}$ & $\mathbf{b} / \mathbf{r}_{\text {petro }}$ \\
\hline $\mathrm{J} 001233.41+010014.2$ & 0.08543 & J001233.34+010010.3 & 1.21324 & 0.16 & 1.11 \\
\hline J021734.23-002637.2 & 0.04069 & J021734.63-002641.9 & 1.55744 & 0.10 & 1.33 \\
\hline J023818.88-003030.5 & 0.03724 & J023819.26-003029.3 & 2.60503 & 0.14 & 0.85 \\
\hline J024329.07+003833.7 & 0.02796 & $\mathrm{~J} 024328.86+003831.2$ & 2.75318 & 0.07 & 0.59 \\
\hline $\mathrm{J} 024421.09+004031.3$ & 0.00932 & $\mathrm{~J} 024420.36+004029.2$ & 2.21791 & 0.09 & 0.42 \\
\hline $\mathrm{J} 032758.83+001652.0$ & 0.03702 & J032759.51+001713.1 & 2.06170 & 0.04 & 0.84 \\
\hline $\mathrm{J} 032803.11+002055.1$ & 0.02363 & $\mathrm{~J} 032801.70+002100.1$ & 0.32205 & 0.02 & 1.91 \\
\hline J075010.54+304106.3 & 0.01477 & $\mathrm{~J} 075010.17+304032.3$ & 1.89210 & 0.07 & 1.82 \\
\hline $\mathrm{J} 112023.20+574429.5$ & 0.00697 & $\mathrm{~J} 112020.12+340555.3$ & 0.76961 & 0.01 & 1.30 \\
\hline $\mathrm{J} 113955.50+132802.0$ & 0.01193 & $\mathrm{~J} 113955.97+132713.3$ & 1.99390 & 0.09 & 1.12 \\
\hline $\mathrm{J} 115115.25+485331.0$ & 0.02564 & $\mathrm{~J} 115118.58+485331.1$ & 1.07180 & 0.11 & 1.18 \\
\hline $\mathrm{J} 122754.83+080525.4$ & 0.00207 & $\mathrm{~J} 122752.60+080526.6$ & 1.62126 & 0.01 & 1.94 \\
\hline $\mathrm{J} 123636.73+141333.1$ & 0.00374 & $\mathrm{~J} 123637.35+141316.2$ & 1.60133 & 0.002 & 1.37 \\
\hline $\mathrm{J} 125700.31+010143.3$ & 0.00930 & $\mathrm{~J} 125703.67+010132.0$ & 0.958967 & 0.04 & 1.18 \\
\hline $\mathrm{J} 131529.74+472958.7$ & 0.00086 & $\mathrm{~J} 131531.57+473054.6$ & 1.72172 & 0.001 & 1.69 \\
\hline $\mathrm{J} 170330.32+240330.8$ & 0.03083 & $\mathrm{~J} 170331.83+240339.8$ & 0.95816 & 0.16 & 1.84 \\
\hline $\mathrm{J} 221216.89+003243.9$ & 0.02971 & $\mathrm{~J} 221217.27+003227.0$ & 2.25410 & 0.08 & 0.99 \\
\hline $\mathrm{J} 233724.00+002330.0$ & 0.00932 & $\mathrm{~J} 233722.01+002238.9$ & 1.37617 & 0.07 & 0.87 \\
\hline
\end{tabular}

Acknowledgements. I thank my department chair, David Turnshek, for approving a travel grant that helped offset some of the cost of my conference participation. Brian Cherinka and I acknowledge the use of SDSS data (see sdss.org/collaboration/credits.html).

\section{References}

Bowen, D. V., Tripp, T. M., \& Jenkins, E. B. 2001, AJ, 121, 1456

Bowen, D. V., Jenkins, E. B., Pettini, M., \& Tripp, T. M. 2005, ApJ, 635, 880

Burkholder, V., Impey, C., \& Sprayberry, D. 2001, AJ, 122, 2318

Deharveng, L., Peña, M., Caplan, J., \& Costero, R. 2000, MNRAS, 311, 329

König, B., Schulte-Ladbeck, R. E., \& Cherinka, B. 2006, AJ, 132, 1844

Haehnelt, M. G., Steinmetz, M., \& Rauch, M. 1998, ApJ, 495, 647

Moos, H. W., et al. 2002, ApJS, 140, 3

Ostriker, J. P. \& Heisler, J. 1984, ApJ, 278, 1

Reimers, D. \& Hagen, H.-J. 1998, A\&SA, 329, L25

Rosenberg, J. L., Bowen, D. V., Tripp, T. M., \& Brinks, E. 2006, AJ, 132, 478

Schulte-Ladbeck, R. E., Rao, S. M., Drozdovsky, I. O., Turnshek, D. A., Nestor, D. B., \& Pettini, M. 2004, ApJ, 600, 613

Schulte-Ladbeck, R. E., König, B., Miller, C. J., Hopkins, A. M., Drozdovsky, I. O., Turnshek, D. A., \& Hopp, U. 2005, ApJL, 625, L79

Searle, L. 1971 ApJ, 168, 327

Williams, R., Jenkins, E. B., Baldwin, J. A., Zhang, Y., Sharpee, B., Pellegrini, E., \& Phillips, M. 2008, ApJ, 677, 1100 